\title{
Prognosis Evaluation of Pulmonary Endarterectomy for Chronic Thromboembolic Pulmonary Hypertension
}

\section{Aissa S*, Mezghani S, Benzarti W, Ben Jazia R, Ben Salem H, Gargouri I, Hayouni A, Garrouche A, Benzarti M and Abdelghani A}

Department of Pneumology, Farhat Hached Hospital, Sousse, Tunisia

\begin{abstract}
Chronic thromboembolic pulmonary hypertension (CTEPH) is one of the leading causes of severe pulmonary hypertension $(\mathrm{PH})$ curable by pulmonary endarterectomy (PEA) necessarily made in the reference centers.

We report the case of a 30-year-old woman with a history of antiphospholipid syndrome diagnosed after a thromboembolic event responsible of her first pregnancy abortion. Two years after this event, she presented a massive and bilateral pulmonary embolism after the delivery of his second pregnancy. She was hospitalized in the intensive care unit and she received thrombolytic treatment relayed by vitamin $\mathrm{K}$ antagonists. One year later, she developed progressive dyspnoea (class III NYHA) and hemoptysis.

Ultrasonic cardiography showed severe pulmonary hypertension (Systolic arterial pulmonary pressure at 120 $\mathrm{mmHg}$ ) and at the right cardiac catheterization (mean arterial pulmonary pressure at $90 \mathrm{mmHg}$ ). Computed tomography pulmonary angiogram and ventilation-perfusion lung scan concluded on CTEPH. Blood gases showed a moderate hypoxemia $(58 \mathrm{mmHg}$ ); conventional treatment was prescribed (oxygen, vitamine $\mathrm{K}$ antagonists and diuretics). The patient was referred to the reference surgical center in Paris and a pulmonary endarterectomy was done. After the surgery, the patient reports an improvement of the class of dsypnea and of the sub-maximal aerobic capacity. The systolic pulmonary pressure decreased at $50 \mathrm{mmHg}$ in the ultrasonic cardiography. The patient actually has a better quality of life and rapidly resumed work.
\end{abstract}

Keywords: Pulmonary hypertension; Chronic thromboembolic pulmonary hypertension; Pulmonary endarterectomy; Antiphospholipid syndrome

\section{Introduction}

Chronic thromboembolic pulmonary hypertension (CTEPH) is one of the causes of severe pulmonary hypertension $(\mathrm{PH})$ and it is responsible of a significant morbidity and mortality $[1,2]$. The CTEPH is essentially a surgical disease, curable by pulmonary endarterectomy (PEA), with acceptable mortality in experienced centers.

The PEA is the curative treatment of choice for CTEPH with restoration of hemodynamics to normal or nearly normal and improvement in clinical symptoms in most patients $[3,4]$.

\section{Case Report}

A 30-year-old woman working a nurse in the department of pediatrics in our hospital, with a history of antiphospholipid syndrome since 2007 diagnosed after a thromboembolic event occurring on his left leg and responsible for her first pregnancy abortion at 12 weeks of amenorrhea.

In 2009, she presented a massive and bilateral pulmonary embolism one month after the delivery of his second pregnancy. She was hospitalized in the intensive care unit and she received thrombolytic treatment relayed by vitamin $\mathrm{K}$ antagonists.

One year later, she developed progressive dyspnea (class III NYHA), hemoptysis and right ventricular failure signs. Utltrasonic cardiography showed severe arterial pulmonary hypertension (Systolic arterial pulmonary pressure [SAPP] at $120 \mathrm{mmHg}$ ) with a dilation of the right cardiac cavities.

Initially, the patient was put under drug therapy associating the vitamin $\mathrm{K}$ antagonist, diuretics and oxygen.

In 2010, the evolution was labeled by progressive worsening of the dyspnea, recurrent hemoptysis and recurrent right cardiac exacerbations.
Chest X-rays showed cardiomegaly and dilation of right cardiac cavities (Figure 1a). Chest tomography angiogram showed pericardial effusion and dilation of the trunk of the pulmonary artery $(40 \mathrm{~mm})$ (Figure 1b) without direct signs of endoluminal thrombi.

Ventilation-perfusion lung scan showed signs of chronic thromboembolic pulmonary hypertension.

Standard blood tests, thyroidien assessment, serologies (HIV, Hepatitis $\mathrm{B} / \mathrm{C}$ ) and coagulation tests were normal.

Blood gases showed moderate hypoxemia (58 $\mathrm{mmHg}$ ) with Pco2 at $38 \mathrm{mmHg}$. The functional tests were not done because of the quality of execution of the exercises.

In the right cardiac catheterization, the mean arterial pulmonary pressure was $90 \mathrm{mmHg}$ and the cardiac Index was $2.81 / \mathrm{min} / \mathrm{m}^{2}$.

All these investigations concluded on the diagnoses of severe chronic post-embolic pulmonary hypertension. The patient was referred to the Surgical center Marie Lannelongue of Paris (France).

In January 2012, the pulmonary endarterectomy (PEA) was realized under extra-corporeal circulation, in deep hypothermia and video-angioscopy with an excellent bilateral disobliteration. The patient had simple post-operative recoveries with a return at home fifteen days

*Corresponding author: Aissa Sana, Assistant Professor of Pneumology, Department of Pneumology, Farhat Hached Hospital, Sousse, Tunisia, Tel: 0021655803301; E-mail: dr.aissa.sana@gmail.com

Received February 25, 2016; Accepted March 15, 2016; Published March 18 2016

Citation: Aissa S, Mezghani S, Benzarti W, Ben Jazia R, Ben Salem H, et al., (2016) Prognosis Evaluation of Pulmonary Endarterectomy for Chronic Thromboembolic Pulmonary Hypertension. J Pulm Respir Med 6: 328. doi:10.4172/2161105X.1000328

Copyright: ( 2016 Aissa S, et al. This is an open-access article distributed under the terms of the Creative Commons Attribution License, which permits unrestricted use, distribution, and reproduction in any medium, provided the original author and source are credited. 
Citation: Aissa S, Mezghani S, Benzarti W, Ben Jazia R, Ben Salem H, et al., (2016) Prognosis Evaluation of Pulmonary Endarterectomy for Chronic Thromboembolic Pulmonary Hypertension. J Pulm Respir Med 6: 328. doi:10.4172/2161-105X.1000328

Page 2 of 3

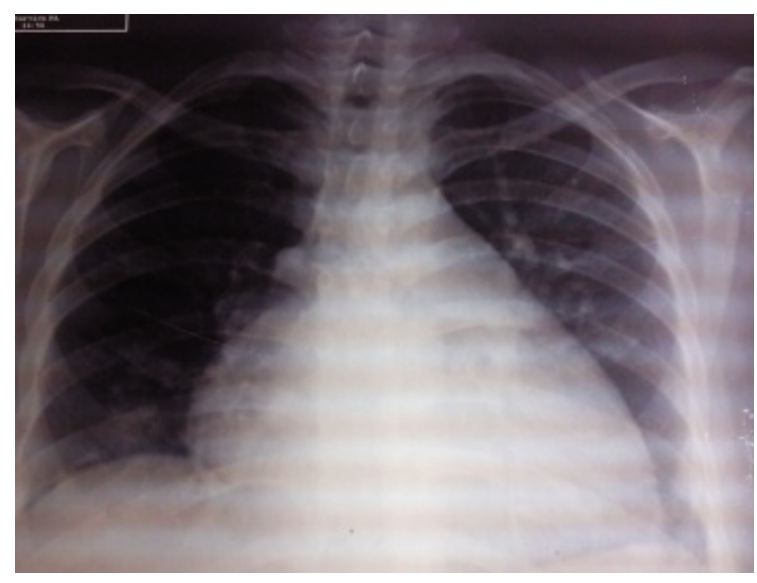

Figure 1a: Chest X-rays: cardiomegaly and dilation of cardiac right cavities.

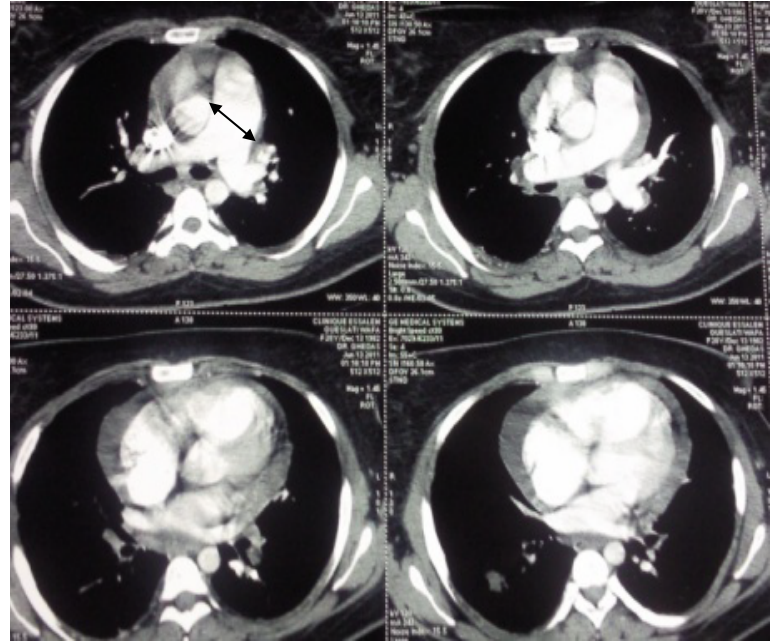

Figure 1b: Chest tomography angiogram (axial plan). Dilation of the trunk of the pulmonary artery $(40 \mathrm{~mm})$ (see arrow). No direct signs of endoluminal thrombi-pericardial effusion.

after the surgery.

The histology of the PEA concluded on an intimal and fibromuscular arterial thickening without any recent thrombosis.

The patient is maintained with vitamin $\mathrm{K}$ antagonist and diuretics.

After six months an evaluation assessment was done:

-The dyspnea passed from class III to class I NYHA.

-The patient has no recurrent haemoptysis.

-The distance walked in the six-minute walking test was 560 meters with one stop at the test and significative oxygen desaturation at 5 points.

-Ultrasonic echocardiography showed a SAPP at $65 \mathrm{mmHg}$ in November 2015.

-Functional tests were normal (spirometry and blood gases).

Our patient resumed work 10 months after surgery.

During the three last years, our patient is doing well and has a better quality of life (Saint George's score: 23\%). His last 6 minute walking test (November 2015) showed a distance walked at 508 meters with oxygen desaturation of 8 points.

\section{Discussion}

Chronic thromboembolic pulmonary hypertension (CTEPH) is a form of pulmonary hypertension $(\mathrm{PH})$ characterized by the persistence of thromboembolic obstructing the pulmonary arteries as an organized tissue and the presence of a variable small vessel arteriopathy [4].

This leads to dyspnoea, and eventually right heart failure and even death. CTEPH is classified as group 4 according to the WHO classification of pulmonary hypertension [5].

CTEPH is the only form of pulmonary hypertension, which is a potentially curable thanks to pulmonary endarterectomy (PEA). However, this is a complicated operation that many patients are not fit enough to undergo and is only of value in patients with proximal forms of the disease. Recent advances in the treatment of CTEPH have shown now that there are three therapeutic options available, with differing levels of success. These include PEA, balloon pulmonary angioplasty (BPA) and medical therapies.

CTEPH is defined as precapillary pulmonary hypertension (mean pulmonary artery pressure $\geq 25 \mathrm{mmHg}$ with a pulmonary capillary wedge pressure $\leq 15 \mathrm{mmHg}$ ) associated with mismatched perfusion defects on ventilation-perfusion lung scan and signs of chronic thromboembolic disease on computed tomography pulmonary angiogram and/or conventional pulmonary angiography, in a patient who received at least 3 months of therapeutic anticoagulation [4]. CTEPH as a direct consequence of symptomatic pulmonary embolism (PE) is rare and should be considered in any patient with unexplained pulmonary hypertension.

Once CTEPH suspected, patients should be addressed to expert centers, where pulmonary angiography, right heart catheterization and high-resolution CT scan will be performed to confirm the diagnosis and to assess the operability. Pulmonary endarterectomy (PEA) remains the gold standard treatment for CTEPH when organized thrombi involve the main, lobar or segmental arteries.

Patients usually present with progressive dyspnoea, which may be associated with lethargy, chest pain, syncope, ankle swelling and haemoptysis. Haemoptysis occurs more frequently in CTEPH (4.8\%) than PAH $(0.6 \%)$ due to bronchial artery dilatation [6]. The diagnosis of CTEPH should be considered in patients with persistent or gradually progressive dyspnea following an acute PE, although CTEPH is rarely diagnosed during regular follow-up after PE. Around 25\% of patients with CTEPH have no history of thromboembolic disease [7]. Thus, CTEPH should be considered in any patient with unexplained $\mathrm{PH}$. Our patient had a history of PE in the context of his antiphospholipid syndrome.

All patients should receive conventional therapy associating diuretics and life-long anticoagulant therapy.

Three therapeutic options are now available in the CTEPH:

\section{Surgical treatment}

The PEA is the gold standard treatment for CTEPH and is associated with improved survival, pulmonary haemodynamics and exercise capacity [7]. This intervention should only be performed by experienced surgeons in specialized centers.

In fact, our patient has improved its haemodynamics parameters, 
Citation: Aissa S, Mezghani S, Benzarti W, Ben Jazia R, Ben Salem H, et al., (2016) Prognosis Evaluation of Pulmonary Endarterectomy for Chronic Thromboembolic Pulmonary Hypertension. J Pulm Respir Med 6: 328. doi:10.4172/2161-105X.1000328

her walked distance in the 6 walking-test and she resumed work 10 months after the surgery.

According to Escribano-Subías et al. PEA patients achieved significantly better hemodynamic and clinical outcomes at one-year follow-up compared to non-PEA patients. Patients not being referred for PEA assessment were older and had a worse functional capacity. Older age was the most deterrent factor for non-operability [8].

\section{Medical treatment}

Small vessel disease in CTEPH has provided the rational for the use of PAH targeted medical therapy in non operable patients with CTEPH or patients with persistent PH after PEA [4].

Riociguat, which is a soluble guanylate cyclase stimulator, has been shown in previous clinical studies to be beneficial in the treatment of chronic thromboembolic pulmonary hypertension. According to Ghofrani et al. we can significantly improve exercise capacity and pulmonary vascular resistance in patients with chronic thromboembolic pulmonary hypertension and this medical approach may be interesting in patients with residual post-operative pulmonary hypertension [9].

Even if our patient has a residual PAH after PEA, the authors found not necessary to add a medical treatment.

\section{Balloon pulmonary angioplasty (BPA)}

Is a new approach therapy for segmental and subsegmental CTEPH. This procedure remains under evaluation [4].

\section{Conclusion}

Our observation illustrates the first successful case of pulmonary endarterectomy proposed for a Tunisian patient. Unfortunately, this intervention is not practiced in Tunisia. Pulmonary endarterectomy (PEA) remains a very expensive surgery in developing countries and the gold standard for the treatment of CTEPH.

\section{Consent}

Written informed consent for publication was obtained from the patient.

\section{Author Contribution}

All authors contributed in the preparation of the manuscript and were involved in the revision of the draft manuscript and have agreed to the final content.

\section{References}

1. McNeil K, Dunning J (2007) Chronic thromboembolic pulmonary hypertension (CTEPH). Heart 93: 1152-1158.

2. Hoeper MM, Mayer E, Simonneau G, Rubin LJ (2006) Chronic thromboembolic pulmo-nary hypertension. Circulation 113: 2011-2020.

3. Galie N, Hoeper MM, Humbert M, Torbicki A, Vachiery JL, et al. (2009) Guidelines for the diagnosis and treatment of pulmonary hypertension: the task force for the diagnosis and treatment of pulmonary hypertension of the European society of cardiology (ESC) and the European respiratory society (ERS), endorsed by the international society of heart and lung transplantation (ISHLT). Eur Heart J 30: 2493-2537.

4. O'Connell C, Montani D, Savale L, Sitbon O, Parent F, et al. (2015) Chronic thromboembolic pulmonary hypertension. La Presse Médicale 44: e409-e416.

5. Kim NH, Delcroix M, Jenkins DP, Channick R, Dartevelle P, et al. (2013) Chronic thromboembolic pulmonary hypertension. J Am Coll Cardiol 62: D92-D99.

6. Lang IM, Simonneau G, Pepke-Zaba JW, Mayer E, Ambrož D, Blanco I, et al. (2013) Factors associated with diagnosis and operability of chronic thromboembolic pulmonary hypertension. A case control study. Thromb Haemost 110: 83-91.

7. Condfiffel R, Kiely DG, Gibbs JS, Corris PA, Peacock AJ, et al. (2009) Pronostic and aetiological factors in chronic thromboembolic pulmonary hypertension. Eur Resp J 33: 332-328.

8. Escribano-Subías P, Del Pozo R, Román-Broto A, Domingo Morera JA, LaraPadrón A, et al. (2016) Management and outcomes in chronic thromboembolic pulmonary hypertension: From expert centers to a nationwide perspective. Int J Cardiol 203: 938-944.

9. Ghofrani HA, D'Armini AM, Grimminger F, Hoeper MM, Jansa P, et al. (2013) Riociguat for the treatment of chronic thromboembolic pulmonary hypertension. N Engl J Med 369: 319-329. 